OPEN ACCESS

Edited by:

Dominic James Hare,

The Florey Institute of Neuroscience and Mental Health, Australia

Reviewed by:

Michael Sperling,

University of Münster, Germany

Andrzej Friedman,

Medical University of Warsaw, Poland

Mark John Hackett,

Curtin University, Australia

*Correspondence:

Asuncion Carmona

acarmona@cenbg.in2p3.fr

Richard Ortega

ortega@cenbg.in2p3.fr

Specialty section:

This article was submitted to

Brain Imaging Methods,

a section of the journal

Frontiers in Neuroscience

Received: 26 June 2019 Accepted: 06 September 2019

Published: 26 September 2019

Citation:

Carmona A, Roudeau S, Perrin L, Carcenac C, Vantelon D, Savasta M

and Ortega $R$ (2019) Mapping Chemical Elements and Iron Oxidation

States in the Substantia Nigra of 6-Hydroxydopamine Lesioned Rats

Using Correlative

Immunohistochemistry With Proton

and Synchrotron Micro-Analysis.

Front. Neurosci. 13:1014.

doi: 10.3389/fnins.2019.01014

\section{Mapping Chemical Elements and Iron Oxidation States in the Substantia Nigra of 6-Hydroxydopamine Lesioned Rats Using Correlative Immunohistochemistry With Proton and Synchrotron Micro-Analysis}

\author{
Asuncion Carmona ${ }^{1,2 *}$, Stéphane Roudeau, ${ }^{1,2}$, Laura Perrin 1,2, Carole Carcenac ${ }^{3,4}$, \\ Delphine Vantelon ${ }^{5}$, Marc Savasta ${ }^{3,4,6}$ and Richard Ortega ${ }^{1,2 *}$
}

${ }^{1}$ UMR 5797, Chemical Imaging and Speciation, CENBG, University of Bordeaux, Gradignan, France, ${ }^{2}$ UMR 5797, CNRS, IN2P3, CENBG, Gradignan, France, ${ }^{3}$ INSERM U1216, Physiopathologie de la Motivation, Grenoble, France, ${ }^{4}$ Grenoble Institute of Neuroscience, Université Grenoble Alpes, Grenoble, France, ${ }^{5}$ SOLEIL Synchrotron, Saint Aubin, France, ${ }^{6}$ Centre Hospitalier Universitaire de Grenoble, Grenoble, France

Brain metal homeostasis is altered in neurodegenerative diseases and the concentration, the localization and/or the chemical speciation of the elements can be modified compared to healthy individuals. These changes are often specific to the brain region affected by the neurodegenerative process. For example, iron concentration is increased in the substantia nigra ( $\mathrm{SN}$ ) of Parkinson's disease patients and iron redox reactions might be involved in the pathogenesis. The identification of the molecular basis behind metal dyshomeostasis in specific brain regions is the subject of intensive research and chemical element imaging methods are particularly useful to address this issue. Among the imaging modalities available, Synchrotron X-ray fluorescence (SXRF) and particle induced X-ray emission (PIXE) using focused micro-beams can inform about the quantitative distribution of metals in specific brain regions. Micro-X-ray absorption near edge spectroscopy (XANES) can in addition identify the chemical species of the elements, in particular their oxidation state. However, in order to bring accurate information about metal changes in specific brain areas, these chemical imaging methods must be correlated to brain tissue histology. We present a methodology to perform chemical element quantitative mapping and speciation on well-identified brain regions using correlative immunohistochemistry. We applied this methodology to the study of an animal model of Parkinson's disease, the 6-hydroxydopamine (6OHDA) lesioned rat. Tyrosine hydroxylase immunohistochemical staining enabled to identify the SN pars compacta (SNpc) and pars reticulata (SNpr) as well as the ventral tegmental area (VTA). Using PIXE we found that iron content was higher respectively in the SNpr > SNpc > VTA, but was not statistically significantly modified by 6OHDA treatment. In addition, micro-SXRF revealed the higher manganese content in the SNpc compared to the SNpr. Using micro-XANES we identified Fe oxidation states in the SNpr and SNpc showing a spectral similarity comparable to ferritin for all 
brain regions and exposure conditions. This study illustrates the capability to correlate immunohistochemistry and chemical element imaging at the brain region level and this protocol can now be widely applied to other studies of metal dyshomeostasis in neurology.

Keywords: brain, imaging, Parkinson, metal, iron, manganese, synchrotron, PIXE

\section{INTRODUCTION}

Metallic elements such as manganese (Mn), iron (Fe), and copper $\mathrm{Cu}$ ) are essential for human since they act as cofactors of many enzymes and participate in cell respiration and metabolism. However, biological essential metals can also be involved in redox reactions leading to the formation of reactive oxygen species (ROS) that promotes cell death. In the nervous system metal dyshomeostasis resulting in production of ROS, protein aggregation or more generally in cell injury may contribute to the etiology of neurodegenerative diseases. It is now well documented that metal dyshomeostasis is involved in the main neurodegenerative disorders, Alzheimer's disease, Parkinson's disease (PD), Huntington's disease, amyotrophic lateral sclerosis and prion diseases (Barnham and Bush, 2014; Collingwood and Davidson, 2014; Ward et al., 2014; Toni et al., 2017). Modifications in metal content and/or their redox species are expected to occur locally in the pathological brains, in the most vulnerable brain regions affected by the pathology. The study of these local modifications requires the use of analytical techniques with microscopic spatial resolution for their observation (Barnham and Bush, 2014; Davies et al., 2014; Collingwood and Adams, 2017; Hackett et al., 2019).

Parkinson's disease is characterized by the progressive loss of dopaminergic neurons in the substantia nigra pars compacta (SNpc) in the ventral midbrain. The etiology of PD is poorly understood and is multifactorial, with inputs from a variety of genetic, environmental and endogen factors, among them Fe dyshomeostasis. In $\mathrm{PD}$, there is a specific increase of $\mathrm{Fe}$ concentration in the $\mathrm{SNpc}$ in comparison to age-matched controls that could be involved in the selective loss of dopaminergic neurons in the SNpc (for review: Sian-Hülsmann et al., 2011; Ward et al., 2014; Liddell and White, 2018). Increased nigral Fe has been proposed as a biomarker for early diagnostic of PD using magnetic resonance imaging (MRI) for Fe quantification in patients (Guan et al., 2017). Some studies have not observed such $\mathrm{Fe}$ increase in the $\mathrm{SN}$ of $\mathrm{PD}$ brains (reviewed in: Friedman and Galazka-Friedman, 2012), reflecting the complexity of this assessment. Another interesting feature about Fe region-specific distribution in the $\mathrm{SN}$ is that Fe content is higher in the SNpr of healthy individuals, the region of the SN that is not affected by neuronal loss in PD (Drayer et al., 1986; Morris and Edwardson, 1994; Morawski et al., 2005). Animal models such as the 6-hydroxydopamine (6-OHDA) lesioned rat have been developed to study PD. 6-OHDA induces a progressive anterograde degeneration of the nigrostriatal pathway, the main anatomical feature in PD. This animal model might be helpful to reproduce some features of Fe dyshomeostasis in $\mathrm{PD}$, in particular the increase of $\mathrm{Fe}$ in the $\mathrm{SNpc}$ (He et al., 1996;
Wang et al., 2004; Hare et al., 2009, 2010; Dexter et al., 2011). For all the above mentioned reasons it is now needed to investigate the distribution of $\mathrm{Fe}$ in specific brain regions using microanalytical methods able to distinguish for instance between the $\mathrm{SNpc}$ and the SNpr thus requiring adequate spatial resolution, especially in animal models where the size of the $\mathrm{SN}$ is smaller than in human brains.

$\mathrm{X}$-ray microanalysis using proton or synchrotron radiation microbeams can be employed to image Fe and other elements content with great sensitivity and quantitative accuracy within local microscopic regions (Ortega et al., 2009; Collingwood and Davidson, 2014; Pushie et al., 2014; Collingwood and Adams, 2017). In addition, micro-XANES can be used to identify Fe(II) and $\mathrm{Fe}$ (III) from differences in their absorption edge energies and pre-edge features (Ortega et al., 2012; Collingwood and Davidson, 2014; Collingwood and Adams, 2017; Porcaro et al., 2018). The determination of chemical element distributions in brain regions requires the use of non-denaturating sample preparation methods usually based on cryogenic protocols of tissue fixation. The classical histology protocols based on chemical fixation and staining of biological tissues are known to disturb the native distribution and speciation of the elements (Chwiej et al., 2005; Ralle and Lutsenko, 2009; Hackett et al., 2011; James et al., 2011; Robison et al., 2012; Davies et al., 2014; Roudeau et al., 2014; Perrin et al., 2015; Jin et al., 2017).

The aim of this study was first to develop a methodology allowing the exact location of the chemical elements in the brain regions of interest ( $\mathrm{SNpc}, \mathrm{SNpr}$, and VTA) while preserving the native element distribution and speciation. This aim was achieved by comparing element imaging to tyrosine hydroxylase (TH) immuno-histochemical staining from adjacent tissue cryosections. Then our second objective was to perform quantitative PIXE analysis and XANES Fe oxidation state speciation in the SN of 6-OHDA lesioned rats at the microscopic level to clearly distinguish between the SNpc SNpr, and VTA regions. We measured the quantitative distribution of Fe and of some other elements ( $\mathrm{P}, \mathrm{S}, \mathrm{Cl}, \mathrm{K}, \mathrm{Ca}, \mathrm{Cu}$, and $\mathrm{Zn}$ ) in each brain area and for the two brain hemispheres, the ipsilateral (IpsiL) 6-OHDA lesioned, and the contralateral (ContraL) non-lesioned side. We also compared the results with those obtained on control animals (sham) injected with a $\mathrm{NaCl}$ solution without 6-OHDA.

\section{MATERIALS AND METHODS}

\section{Animal Experiments}

Animal experiments were carried out according to the guidelines for the care and use of laboratory animals approved by the 
European Community Council Directive of 24 November 1986 (86/609/EEC), and the ethics committee of the Grenoble Institute of Neuroscience. One month old Wistar rats were anesthetized with a mixture of xylazine (15 mg/kg, i.p.) and ketamine (100 mg/kg, i.p.) $30 \mathrm{~min}$ before intracraneal 6-OHDA injection (6-Hydroxydopamine hydrobromide, H116, Sigma). Rats were secured in a Kopf stereotaxic apparatus (Phymep, Paris, France) and $1 \mu \mathrm{L}$ of 6-OHDA (injured animals) or $1 \mu \mathrm{l}$ of sterile $0.9 \% \mathrm{NaCl}$ (sham conditions), were unilaterally injected in the $\mathrm{SNpc}$ at a flow rate of $0.5 \mu \mathrm{L} / \mathrm{min}$. Two weeks later rats were anesthetized by inhalation of isoflurane and the dorsal skull was exposed. Brains were perfused to avoid vascularization. In order to preserve structure and chemical composition after extraction, brains were cryofixed by rapid plunging into a cooled cryogenic liquid (isopentane cooled with dry ice at $-40^{\circ} \mathrm{C}$ ), and then stored at $-80^{\circ} \mathrm{C}$. The correct location of the injection cannula was checked by collecting the SN using the atlas of Paxinos and Watson (2007). Both sides of the brain were studied, the ipsilateral (IpsiL, injured side) and the contralateral (ContraL, control side). Samples were obtained from 15 6-OHDA lesioned animals and 12 sham animals. 6-OHDA administration resulted in a $35-85 \%$ (mean $60 \%$ ) range decrease in $\mathrm{TH}$ expression as measured by TH immuno-histochemistry.

\section{Sample Preparation: Overview}

Our aim was to determine the elemental content in specific regions of the rat brain: $\mathrm{SNpc}, \mathrm{SNpr}$ and VTA. The location of these specific regions in the brain can be performed by immunohistochemical staining of the dopamine synthesis enzyme $\mathrm{TH}$, as explained in the next section. However, immunohistochemistry preparations based on chemical fixation and staining are not suited for chemical imaging since they are known to alter elemental distribution and speciation (Chwiej et al., 2005; Ralle and Lutsenko, 2009; Hackett et al., 2011; James et al., 2011; Robison et al., 2012; Davies et al., 2014; Roudeau et al., 2014; Perrin et al., 2015; Jin et al., 2017). To make both purposes compatible we used cryofixed animal brains and performed histological staining and chemical analysis on consecutive, adjacent, brain tissue cryo-sections. By superposition of both cryo-sections we could determine the areas of interest, in this case the localization of SNpr, SNpc, and VTA, in the unstained sections used for X-ray microanalysis. In order to identify precisely the brain regions of interest several important steps need to be considered before sectioning. The workflow of the procedure is illustrated in Figure 1.

Two adjacent cryo-sections are cut: one for the elemental analysis (PIXE, XANES, or SXRF) and the other one for histological staining. Cryo-sections for histological staining are deposited onto usual microscope slides. For PIXE, XANES, or SXRF analyses, glass slides cannot be used since they contain element impurities and thus an appropriate sample holder must be chosen. Several materials are available such as silicon nitride membranes or thin polymers films of ultrapure quality (Perrin et al., 2015). In this study we used a polycarbonate film of $2 \mu \mathrm{m}$ thickness stretched on a plastic frame holder made of PEEK (Figure 2A). Both brain cryo-sections must have a common face to perform correlative imaging. This is the face that is cut by

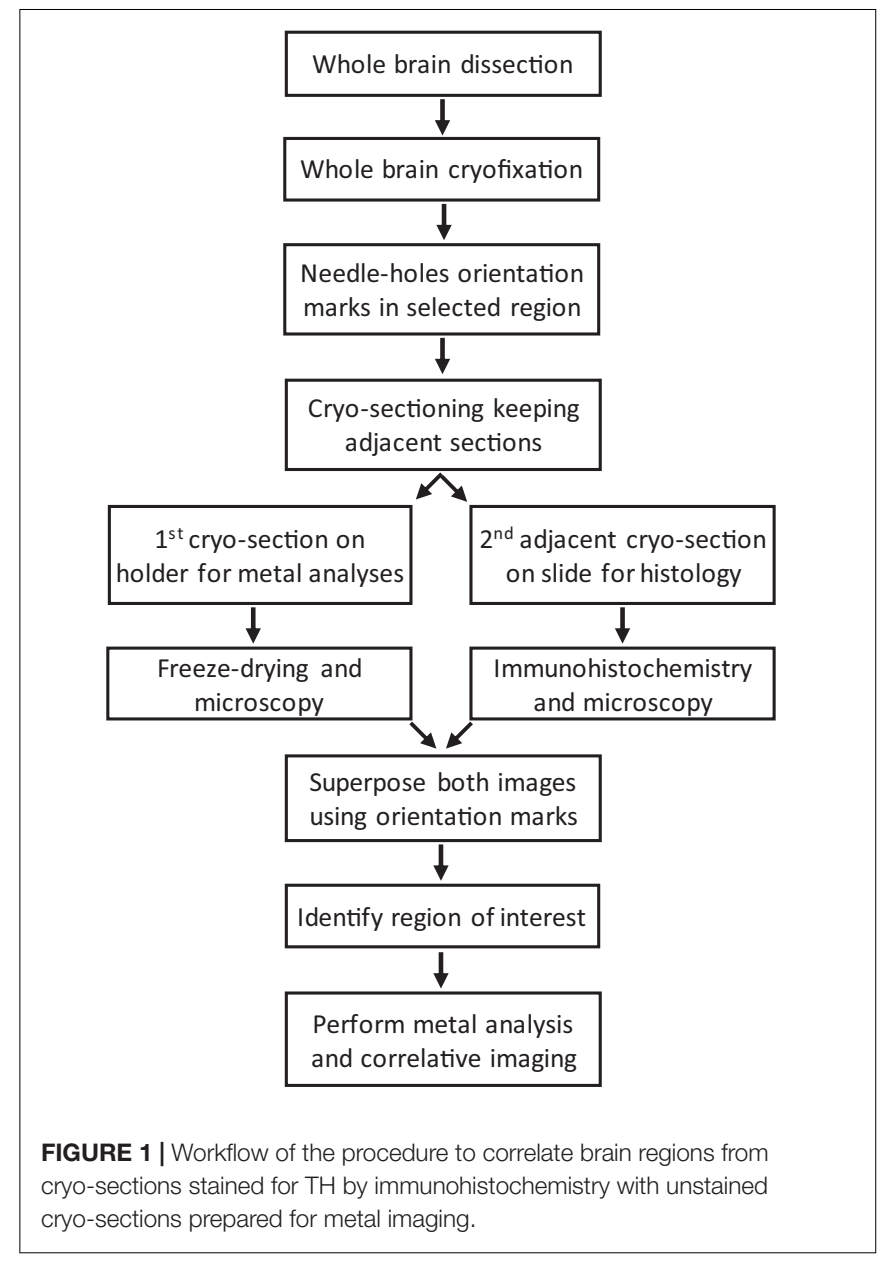

the knife when performing the first cryo-section as highlighted in red in Figure 2B. This face should be deposited top up on the sample holders, the PEEK/polycarbonate sample holder and the microscope slide, as illustrated in Figure 2C. To superimpose precisely the images of the two adjacent cryo-sections, we used orientation marks consisting in some small pinholes made with a needle in the tissue before sectioning. By superposition of the holes, the region of interest can be identified on both cryosections, as schematically shown in Figure 2C.

\section{Preparation of Brain Cryo-Sections}

The frozen tissue block was transferred into the cryo-microtome and mounted with tissue freezing medium for cryo-sectioning at $-25^{\circ} \mathrm{C}$. For PIXE, XANES, and SXRF analyses semi-thin $50 \mu \mathrm{m}$ cryo-sections of brain tissues were prepared. If the tissue section is too thin, the metal content might not be detectable or leading to too long acquisition times to perform the experiments. If the tissue section is too thick then some metal contribution could come from deeper brain regions below the region of interest. The polycarbonate film on the PEEK sample holder was placed into the cryostat at $-25^{\circ} \mathrm{C}$ to cool down before to deposit the cryo-section. Once the tissue and the sample holder reach the right temperature, the cryo-section is deposited onto the polycarbonate/PEEK sample holder, keeping the face to be 


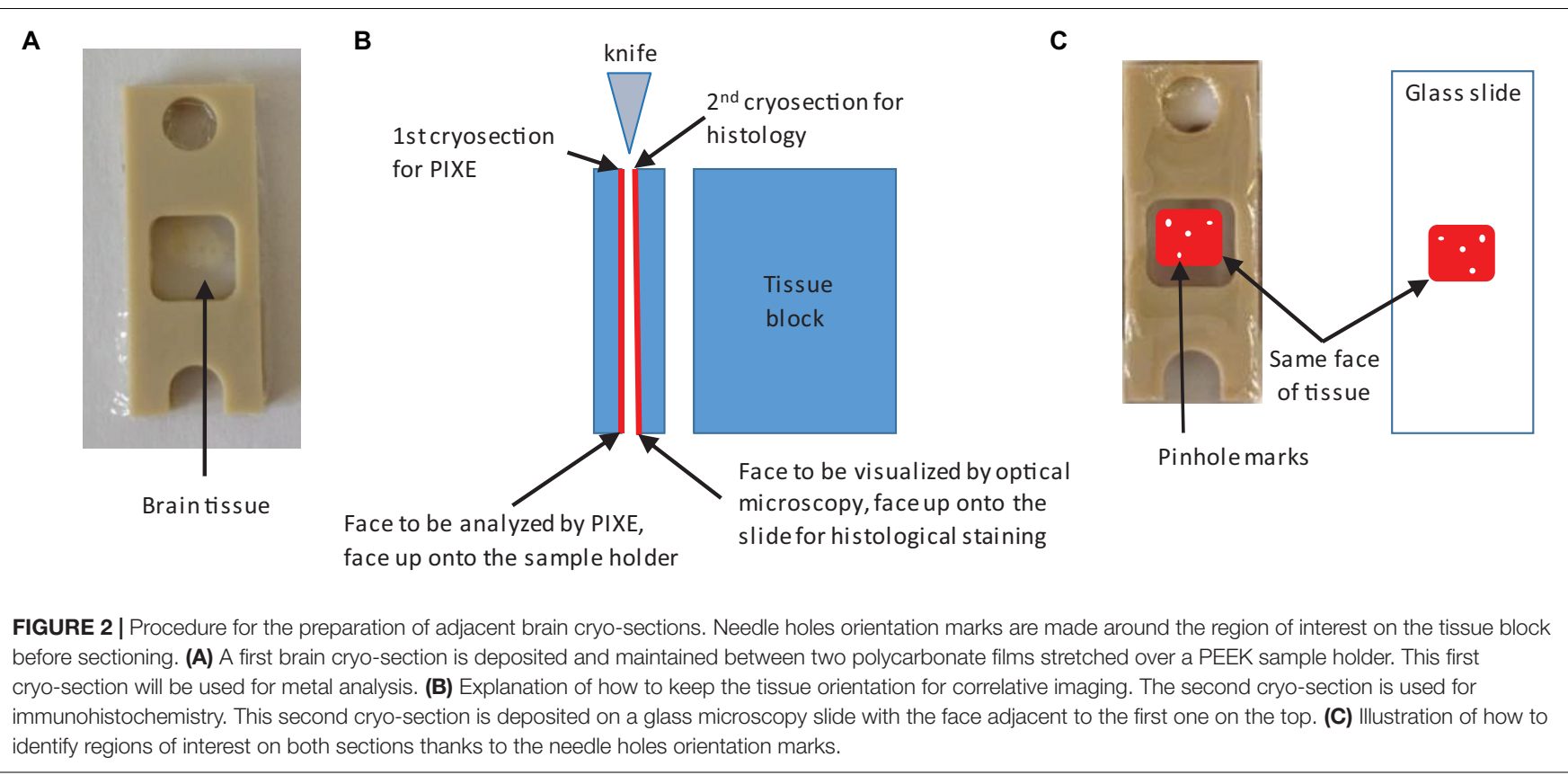

analyzed up. To avoid tissue deformation, the cryo-section is covered with a second PEEK/polycarbonate sample holder. By this mean, the brain cryo-section is maintained between the two polycarbonate films in a stable position during handling and analysis. A second $20 \mu \mathrm{m}$ thickness cryo-section immediately adjacent to the previous one is placed onto a microscope glass slide for histological staining. Similarly, to the previous step, the slide is placed into the cryostat to reach the correct temperature, and the brain cryo-section is deposited keeping the common face up (Figure 2).

Depending on the analytical method that will be further used, the initial $50 \mu \mathrm{m}$ cryo-sections prepared for elemental analysis are either freeze dried for PIXE analysis, in order to be placed under vacuum, or stored in cryotubes placed in liquid nitrogen until XANES and SXRF analyses which are performed with a sample cryostage on frozen hydrated samples. For PIXE analysis, cryo-sections were freeze dried with a Christ alpha 2-4 LD plus at $-85^{\circ} \mathrm{C}$ under vacuum $\left(2.5 \times 10^{-3} \mathrm{mbar}\right)$ during $24 \mathrm{~h}$ and slowly rewarmed to room temperature under vacuum.

\section{TH Immuno-Histochemical Staining}

Brain tissue sections from the whole brain, IpsiL and ContraL sides, were mounted on silane-coated microscope slides. Tissue sections were postfixed in $4 \%$ paraformaldehyde, thoroughly twice washed with Tris buffered-saline (TBS, $0.1 \mathrm{M}, \mathrm{pH}$ 7.4) and incubated for $1 \mathrm{~h}$ in $0.3 \%$ Triton $\mathrm{X}-100$ in TBS (TBST) and 3\% normal goat serum (NGS, Sigma-Aldrich, St. Quentin Fallavier, France) at room temperature. They were then incubated with primary antisera diluted in TBST supplemented with $1 \%$ NGS overnight, at $4^{\circ} \mathrm{C}$. The antiserum was diluted 1:500 for $\mathrm{TH}$ staining (mouse monoclonal antibody; Chemicon, Temecula, CA, United States). Antibody binding was detected with avidin-biotin-peroxidase conjugate (Vectastain
ABC Elite, Vector Laboratories, Burlingame, CA, United States), with 3,3'-diaminobenzidine as the chromagen. The detection reaction was allowed to proceed for 1-3 min. Sections were dehydrated in a series of graded ethanol solutions, cleared in xylene, mounted in DPX (DBH Laboratories Supplies, Poole, United Kingdom) and covered with a coverslip for microscopy (Favier et al., 2013).

\section{Identification of Brain Regions}

Tyrosine hydroxylase immuno-labeled and unstained adjacent freeze dried sections are observed by optical transmission microscopy and the images of the whole sections are reconstructed. Superposition of the histological image reconstruction and of the optical microscopy image of the freeze dried sample are obtained by superposition of the pinhole orientation markers made before sectioning. The areas of interest in the immuno-histochemical section are used to determine the localization of these areas in the freeze dried sections. The overall procedure for the superposition of histological images and optical microscopy images of freeze dried samples is illustrated in Figure 3.

\section{Micro-PIXE Analyses}

Micro-PIXE analyses were performed at CENBG using a 3.5 MV Singletron particle accelerator (HVEE, Netherlands) at AIFIRA facility (Applications Interdisciplinaires des Faisceaux d'Ions en Région Aquitaine). Micro-PIXE and micro-RBS (Rutherford Backscattering Spectrometry) analyses were carried out simultaneously, using the high resolution microbeam line, with a proton beam of $3.0 \mathrm{MeV}$, focused at $2 \mu \mathrm{m}$ spatial resolution and resulting in a $0.8 \mathrm{nA}$ beam current. Emitted X-rays were collected using two lithium drifted silicon X-ray detectors (Sirius, United Kingdom), placed at 
A

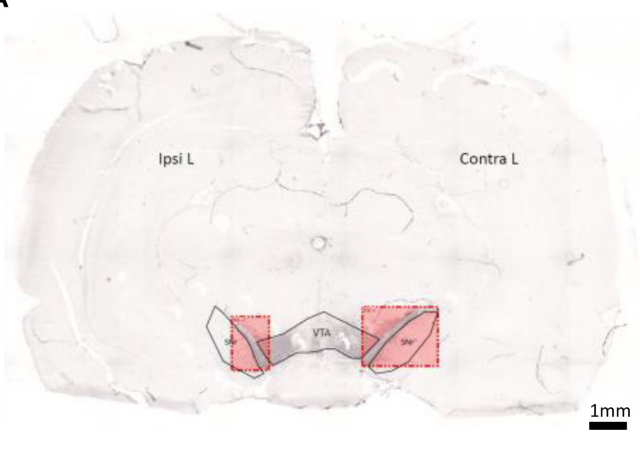

B

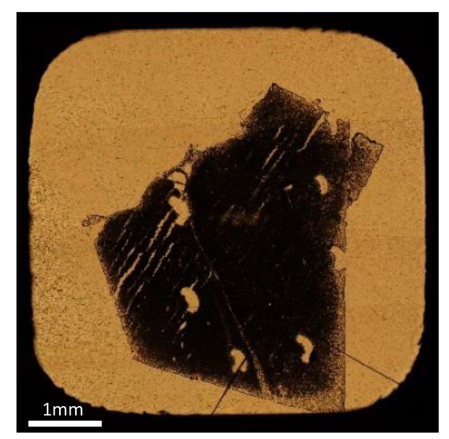

C

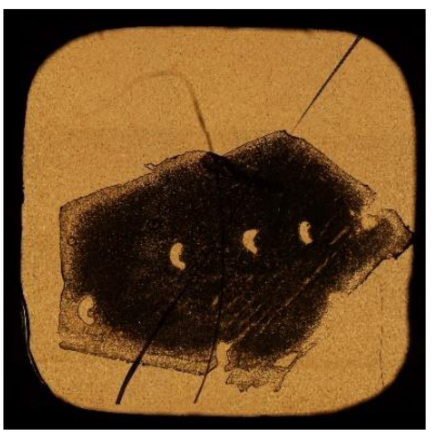

D

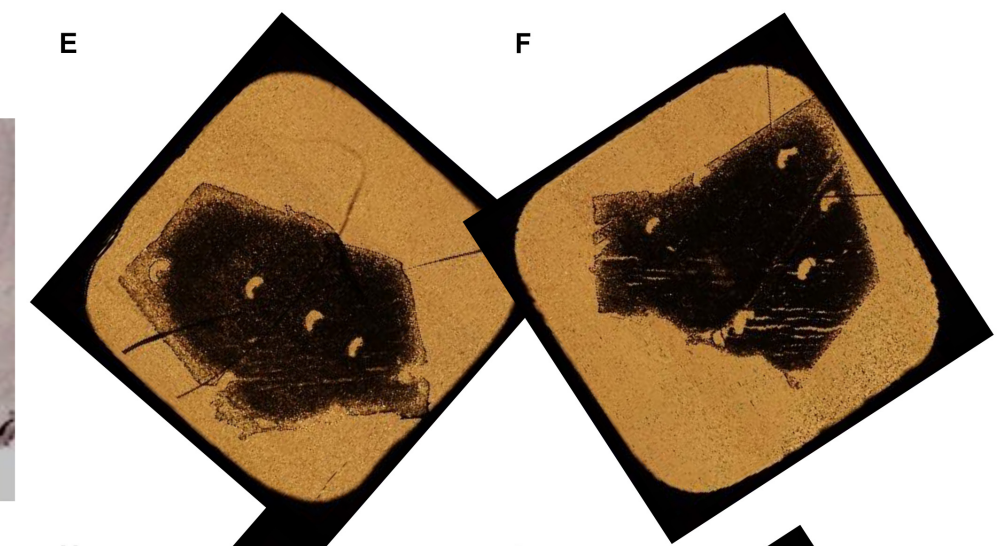

E

E $F$

F
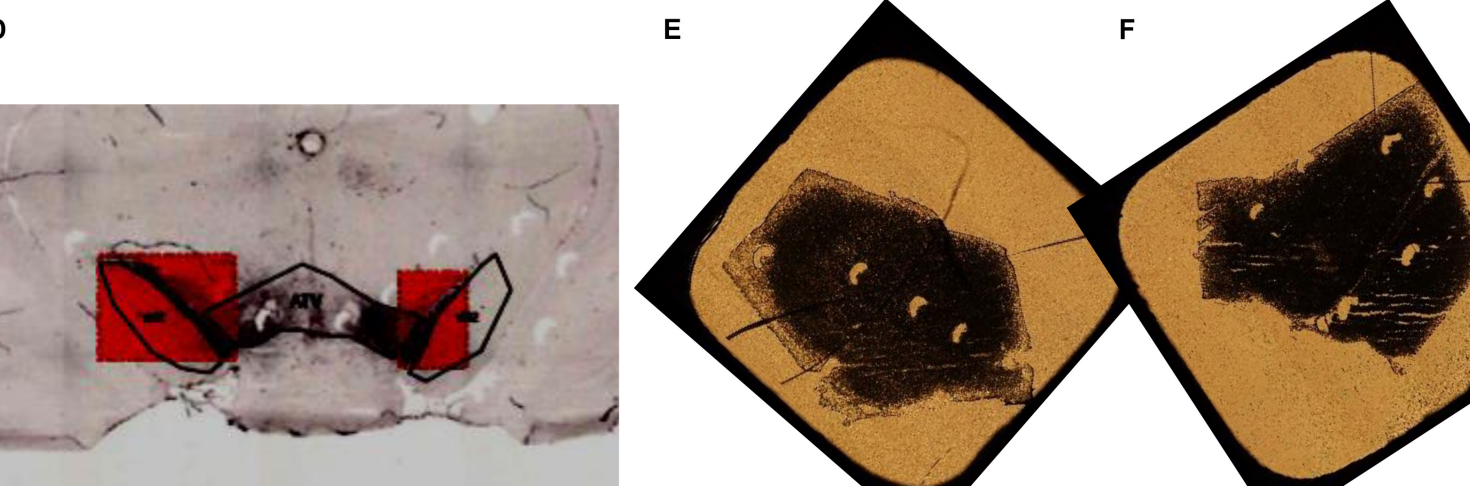

G

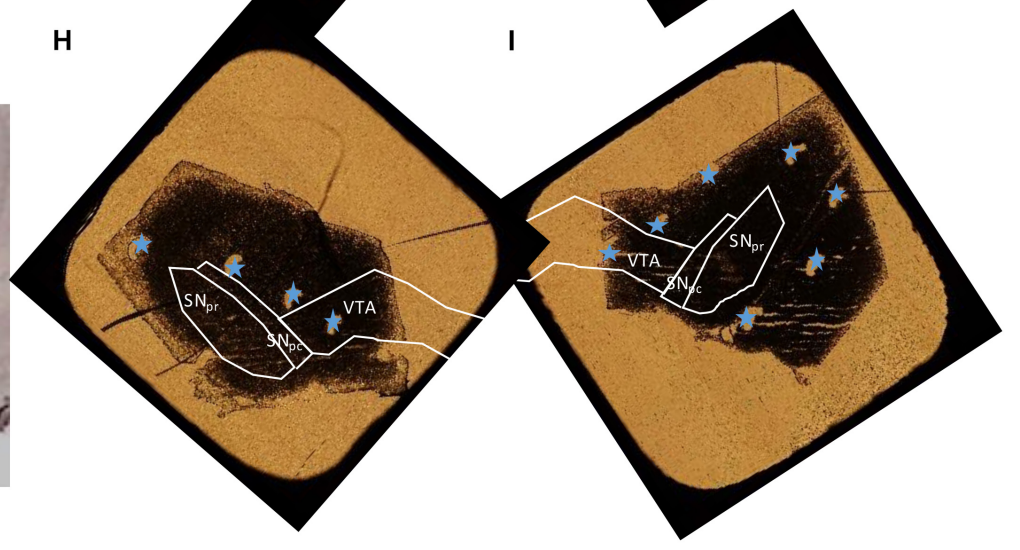

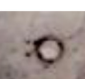

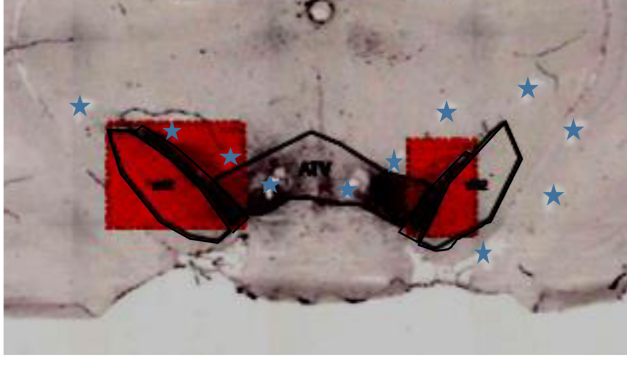

$\mathrm{H}$

FIGURE 3 | Overall procedure for superposition of TH immuno-histochemical sections and the adjacent freeze dried unstained section for PIXE analysis. (A) Entire brain section stained with TH to localize the dopaminergic pathway, SNpc, SNpr, and VTA. (B,C) Optical transmission microscopy images of freeze dried sections for PIXE analyses (B: IpsiL brain side and C: ContraL brain side). (D,G) Zoom of the stained region, to distinguish the pinholes done around the SN (indicated with blue stars). These images have a horizontal flip with respect to the initial image in order to obtain the same orientation for the pinholes than on freeze dried samples. (E,F) PIXE section images, applying a rotation, to display the same orientation of pinholes than on the immuno-histochemical sections. (H,I) Correctly oriented images using the pinholes (marked with a blue star) to locate precisely the SNpc, SNpr, and VTA regions on the sections for PIXE analysis. Scale bars $=1 \mathrm{~mm}$.

$135^{\circ}$ on both sides of the beam axis direction. Backscattered particles were collected using a passivated implanted planar silicon detector (Canberra, France) placed at $135^{\circ}$ below the incident beam direction.

The microbeam setup for $3 \mathrm{MeV}$ protons allows to scan square surfaces of maximum $750 \mu \mathrm{m}$ side. Therefore, for each tissue section, we performed several analyses in order to cover all the surface of the regions of interest, $\mathrm{SNpc}, \mathrm{SNpr}$, and VTA, as previously described for the analysis of large tissue sections (Carmona et al., 2017). Thanks to the superposition of the images of $\mathrm{TH}$ staining and of the adjacent freeze dried section, we could exactly delimit the regions of interest to be analyzed by PIXE (Figure 3). Then PIXE and RBS local spectra corresponding exactly to the brain regions of interest (SNpr, SNpc, VTA) were extracted post-analysis from each of the individual $750 \mu \mathrm{m} \times 750 \mu \mathrm{m}$ scan. Element concentration expressed in $\mu \mathrm{g} \cdot \mathrm{g}^{-1}$ were determined for each of the selected regions. Finally, the elemental content in the three regions of 
interest (SNpc, SNpr and VTA) was calculated as the mean value of the extracted regions of all the individual scans performed on each region. The number of analyzed animals was 15 for the 6-OHDA group and 12 for the Sham group.

Quantitative data treatment of PIXE spectra was performed using the GUPIXWIN software (Campbell et al., 2010). This software allows determining the elemental areal mass $\left(\mu \mathrm{g} \cdot \mathrm{cm}^{-2}\right)$. Charge normalization of PIXE data was obtained by fitting RBS spectra with SINMRA software (Mayer, 1997) following a methodology described in earlier publications (Carmona et al., 2008, 2017; Perrin et al., 2015). The areal mass of the tissues $\left(\mathrm{g} \cdot \mathrm{cm}^{-2}\right)$ was also determined by RBS, this parameter is necessary to normalize the PIXE areal mass and express the element content as $\mu \mathrm{g}$ of element per $\mathrm{g}$ of dry mass $\left(\mu \mathrm{g} \cdot \mathrm{g}^{-1}\right)$. Calibration of the X-ray detectors for quantitative analysis was performed using Micromatter ${ }^{\mathrm{TM}}$ XRF calibration standards.

\section{Micro XANES and SXRF Analyses}

Micro-XANES and micro-SXRF experiments were conducted at SOLEIL, LUCIA beamline (Flank et al., 2006; Vantelon et al., 2016). The beam was focused to $4.0 \mu \mathrm{m} \times 2.5 \mu \mathrm{m}$ by means of mirrors installed in a Kickpatrick-Baez. Micro-XANES analyses were performed at Fe K-edge absorption energy, where the provided flux was $6.4 \times 10^{9} \mathrm{ph} \cdot \mathrm{s}^{-1}$. The spectra were acquired in fluorescence mode by scanning the near edge region (7050$7250 \mathrm{eV})$ at a rate of $10 \mathrm{~s}$ per energy step with a $0.2 \mathrm{eV}$ energy step around the edge (7100-7160 eV), $2 \mathrm{eV}$ below the edge (7050$7100 \mathrm{eV}$ ), and $1 \mathrm{eV}$ above the edge (7160-7250 eV). Experiments were conducted on frozen hydrated tissue sections. Samples were maintained in a dried nitrogen atmosphere before the experiment and cooled by thermal contact with the cryo-stage $\left(-196^{\circ} \mathrm{C}\right)$ during the measurements. The samples were prepared in the same way than for PIXE analyses but without freeze drying, and were kept refrigerated at all time in liquid nitrogen. Iron oxidation state in the SNpc and SNpr for 6-OHDA injured and sham animals were measured at least in triplicate. XANES spectra were calibrated in energy against the absorption edge position of $\mathrm{Fe}(0)$ metallic foil, whose first inflection point is $7112 \mathrm{eV}$. Three different reference compounds were analyzed: $\mathrm{FeO}$ as $\mathrm{Fe}(\mathrm{II})$ standard, $\mathrm{Fe}_{2} \mathrm{O}_{3}$ and ferritin as $\mathrm{Fe}$ (III) standards. Micro-SXRF imaging was performed after micro-XANES analyses. The energy was fixed at $7200 \mathrm{eV}$, the pixel resolution was $4 \mu \mathrm{m}$ and the time of analyses par pixel was set to $1 \mathrm{~ms}$.

\section{Statistical Analyses}

Statistical analyses were conducted using open access R software (R Core Team, 2013), R Commander (Fox and Bouchet-Valat, 2018), and RStudio (RStudio Team, 2016). We compared each brain region ( $\mathrm{SNpc}, \mathrm{SNpr}$, and VTA) and brain side (ContraL and IpsiL) for the two injection conditions (sham and 6OHDA injured). The normality postulate was not always fulfilled and we considered the regions and brain sides as not paired. Multiples comparison of elemental content were performed using Kruskal Wallis test. Wilcoxon comparison as post hoc test was used to determine, for each element, which zone show significant differences, no method to adjust $p$-value was used (Feise, 2002).

\section{RESULTS}

\section{Element Content in Brain Regions Measured by Micro-PIXE}

Particle induced X-ray emission technique, in combination with RBS, enables the quantification of the elemental content in biological tissues (Carmona et al., 2008, 2017; Perrin et al., 2015). We could determine $\mathrm{P}, \mathrm{S}, \mathrm{Cl}, \mathrm{K}, \mathrm{Ca}, \mathrm{Fe}, \mathrm{Cu}$, and $\mathrm{Zn}$ in freeze dried brain tissues sections. We analyzed 15 animals injured with 6-OHDA and 12 control animals (sham, injected with a $\mathrm{NaCl}$ saline solution). The injection was unilateral, but both sides of the brain, IpsiL and ContraL, were analyzed. Three dopaminergic zones were distinguished in each side, SNpc, SNpr, and VTA. The quantitative results are presented in Figure 4 showing boxplots with the elemental concentrations, expressed in $\mu \mathrm{g} \cdot \mathrm{g}^{-1}$, for each brain region, for injured and control animals (6-OHDA and Sham, respectively), and in each side of the brain (ContraL and IpsiL).

When comparing the sides and regions of interest between lesioned animals and controls, Fe concentrations are unchanged (Figure 4). This result indicates that the injection of 6-OHDA did not significantly modified the Fe content in the SNpc, SNpr, and VTA of the lesioned animals despite a in a 35-85\% (mean 60\%) range decrease in $\mathrm{TH}$ expression. On the other hand, Fe content was found statistically significantly higher in the SNpr than in the SNpc for each brain side and for all conditions (Figure 4). Hence, we found that Fe content is well compartmentalized in the three dopaminergic regions, the lowest values were found in the VTA and the highest in SNpr. Statistically significant differences were also observed for sulfur, with a lower content in the VTA than in SNpc or SNpr in both 6-OHDA and Sham cases. Except for iron and sulfur no other important significant differences were noticed.

\section{Iron Oxidation State Measured by Micro-XANES and XRF Imaging of Fe and $\mathrm{Mn}$}

Iron can be present in biological tissues in different oxidation states, mainly $\mathrm{Fe}(\mathrm{II})$ and $\mathrm{Fe}(\mathrm{III})$, which determine the reactivity and potential toxicity of this element. Iron oxidation state was measured in the SNpc and the SNpr and for both brain sides (IpsiL and ContraL) and injection conditions (6-OHDA and sham). Micro-XANES data indicated that Fe is present in form of $\mathrm{Fe}(\mathrm{III})$, in all cases, regardless of the injection side or the brain region (Figure 5 and Table 1). Micro-XANES spectra for all brain regions show that Fe exhibits very similar shapes and values of the first derivative inflection point as for the ferritin standard, suggesting that iron could be complexed principally to ferritin (Figure 5 and Table 1).

Following micro-XANES analyses, micro-SXRF element mapping were performed on the same samples. The objective was to map Fe and $\mathrm{Mn}$ distribution at the junction of SNpc 


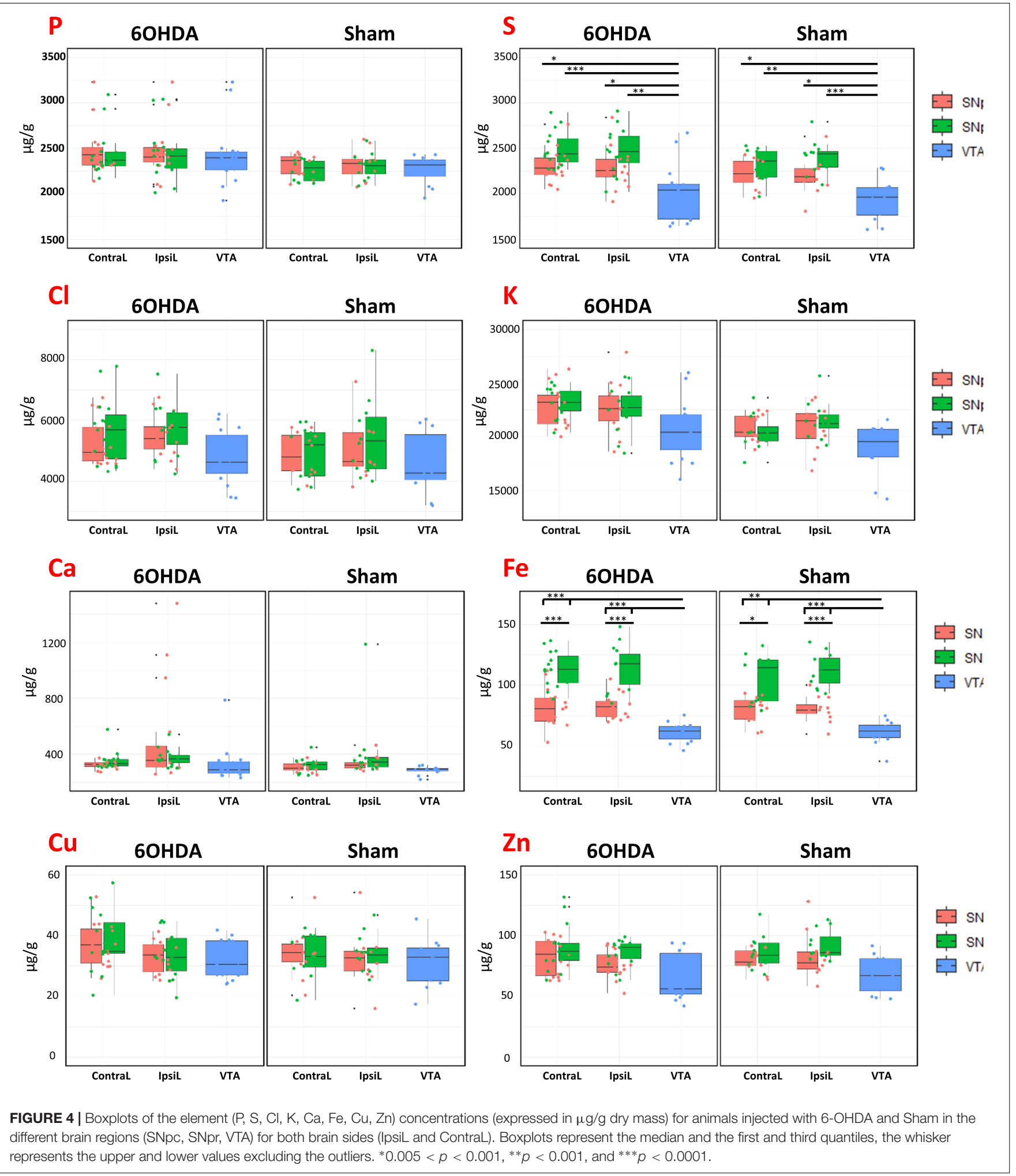

and SNpr. Mn was below the limit of detection for microPIXE analysis but could be detected by SXRF which is a more sensitive analytical method (Ortega et al., 2009). Similarly, to
micro-PIXE results, micro-SXRF analyses confirmed the higher content of Fe in the SNpr vs. the SNpc (Figure 6). Contrary to $\mathrm{Fe}$ distribution, $\mathrm{Mn}$ intensity is higher in the SNpc than in the 


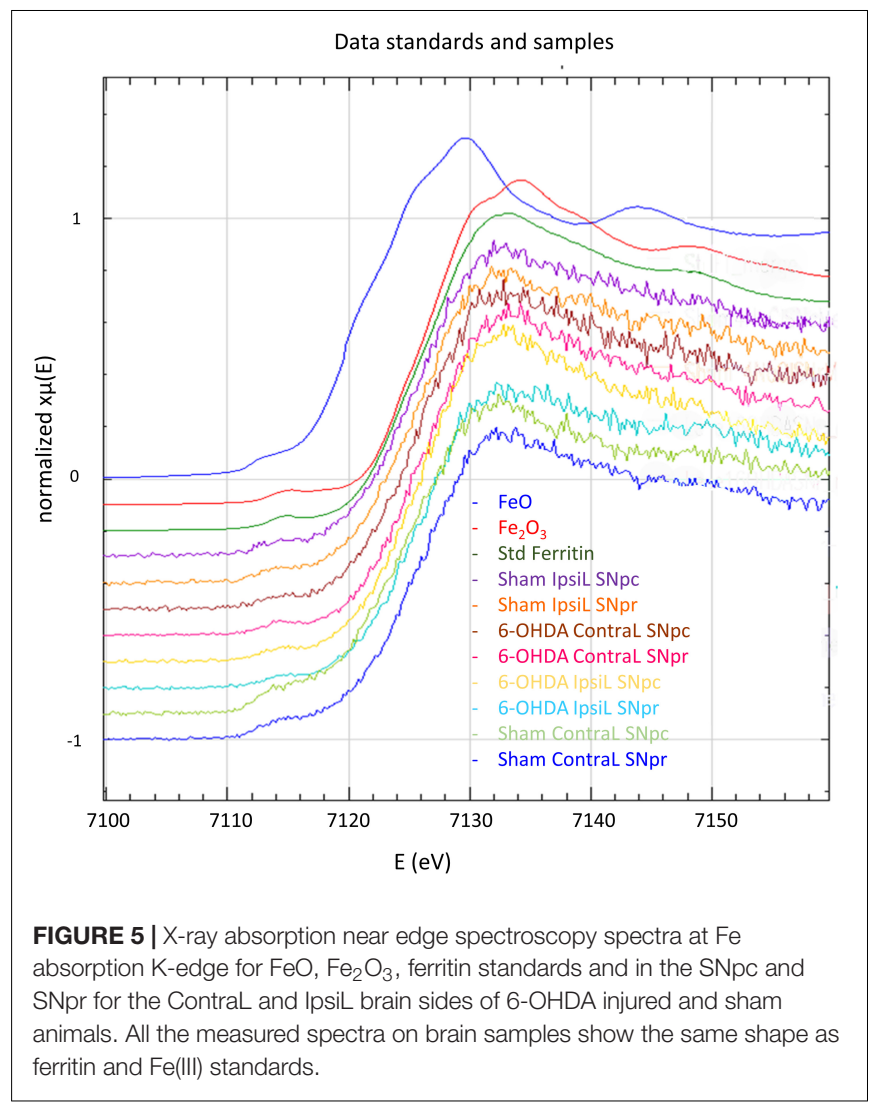

SNpr. This preferential Mn accumulation in the SNpc appears in sham and 6-OHDA injured animals and in both brain sides, IpsiL and ContraL.

\section{DISCUSSION}

Micro-analytical and spectroscopic imaging methods are required to quantify and probe oxidation states of metals in local areas of the brain. This need is particularly well illustrated in

TABLE 1 | Values of the XANES inflection point of the first derivative for each brain region analyzed, the inflection occurs at the same value as $\mathrm{Fe}(\mathrm{III})$ and ferritin standards and for all brain regions and injection conditions.

\begin{tabular}{lc}
\hline Sample & EO (eV) \\
\hline Standard Fe(II) & $7119.8 \pm 0.5$ \\
Standard Fe(III) & $7123.6 \pm 0.5$ \\
Ferritin & $7123.9 \pm 0.5$ \\
Sham Ipsilateral SNpc & $7123.3 \pm 0.5$ \\
Sham Ipsilateral SNpr & $7123.4 \pm 0.5$ \\
Sham Contralateral SNpc & $7124.2 \pm 0.5$ \\
Sham Contralateral SNpr & $7123.3 \pm 0.5$ \\
60HDA Ipsilateral SNpc & $7123.9 \pm 0.5$ \\
60HDA Ipsilateral SNpr & $7124.3 \pm 0.5$ \\
60HDA Contralateral SNpc & $7124.0 \pm 0.5$ \\
60HDA Contralateral SNpr & $7123.8 \pm 0.5$
\end{tabular}

the case of the $\mathrm{SN}$ that is separated in two regions, $\mathrm{SNpc}$ and $\mathrm{SNpr}$, with distinct $\mathrm{Fe}$ content. Iron histochemistry revealed that non-haem Fe staining was higher in the SNpr than in the SNpc in the human brain (Drayer et al., 1986; Morris and Edwardson, 1994). This result was further confirmed for total $\mathrm{Fe}$ using quantitative micro-PIXE measurements (Morawski et al., 2005). This Fe compartmentalization in the SN was also observed in mice using immuno-assisted laser ablation-inductively coupled plasma-mass spectrometry (Hare et al., 2014), or using a combination of LA-ICPMS and SXRF (Davies et al., 2015). Our results on Wistar rats are in agreement with these studies showing that that $\mathrm{Fe}$ content is higher in the SNpr than in SNpc and higher in the $\mathrm{SNpc}$ than in VTA. These results reproduce well the data obtained in human (Drayer et al., 1986; Morris and Edwardson, 1994; Morawski et al., 2005) and in mice (Davies et al., 2015), including the comparison with the adjacent VTA region (Hare et al., 2014). The higher Fe content in the SNpr vs. SNpc could be due to the presence of oligodendrocytes which are cells known to contain more Fe than neurons (Snyder and Connor, 2009).

Anomalous $\mathrm{Fe}$ handling has been proposed to be involved in the selective loss of dopaminergic neurons from the SNpc (Sian-Hülsmann et al., 2011; Ward et al., 2014). Using Fe histochemical assays, an increase of $\mathrm{Fe}$ has been reported in the SNpc from PD patients (Sofic et al., 1988; Riederer et al., 1989; Morris and Edwardson, 1994; Faucheux et al., 2003) which might contribute to ROS production and neuronal degeneration. However, the quantitative distribution and speciation of $\mathrm{Fe}$ in the $\mathrm{SN}$ relied mostly on semi-quantitative Fe histochemical methods, and these methods are not sensitive to all chemical species of the metal. Electron X-ray microanalysis confirmed $\mathrm{Fe}$ dyshomeostasis with the observation of increased $\mathrm{Fe}$ in the neurons from the SN of PD patients (Oakley et al., 2007), as well as SXRF showing the global increase of Fe in the SN of PD patients (Popescu et al., 2009), and in neuromelanin neurons (Davies et al., 2014). The 6-OHDA rat model has been suggested as an animal model to study Fe dyshomeostasis in PD. For instance, this animal model has been successfully used to study the effect of $\mathrm{Fe}$ chelation on the protection of dopaminergic neurons from the $\mathrm{SNpc}$ showing a neuroprotective effect of clinically available Fe chelators (Dexter et al., 2011). Initial observations using histochemical Fe staining have shown that the degeneration of nigrostriatal dopaminergic neurons following 6-OHDA lesions is associated with increased $\mathrm{Fe}$ accumulation in the $\mathrm{SN}$ of about $35 \%$ in the IpsiL SN vs. the ContraL SN (Oestreicher et al., 1994). This result was confirmed by a quantitative spectrometric approach based on micro-PIXE analysis showing a $26 \%$ increase of Fe in the SN from the 6OHDA IpsiL lesioned-side of the animals (Watt et al., 1995). More recently, the use of quantitative LA-ICP-MS (laser ablationinductively coupled plasma-mass spectrometry) also showed a $20 \%$ increase of $\mathrm{Fe}$ in the SN from the IpsL lesioned-side (Hare et al., 2010).

In our study, however, we did not observe any variation of Fe content following 6-OHDA treatment, in any of the studied brain regions (SNpc, SNpr, and VTA) (Figure 4). 

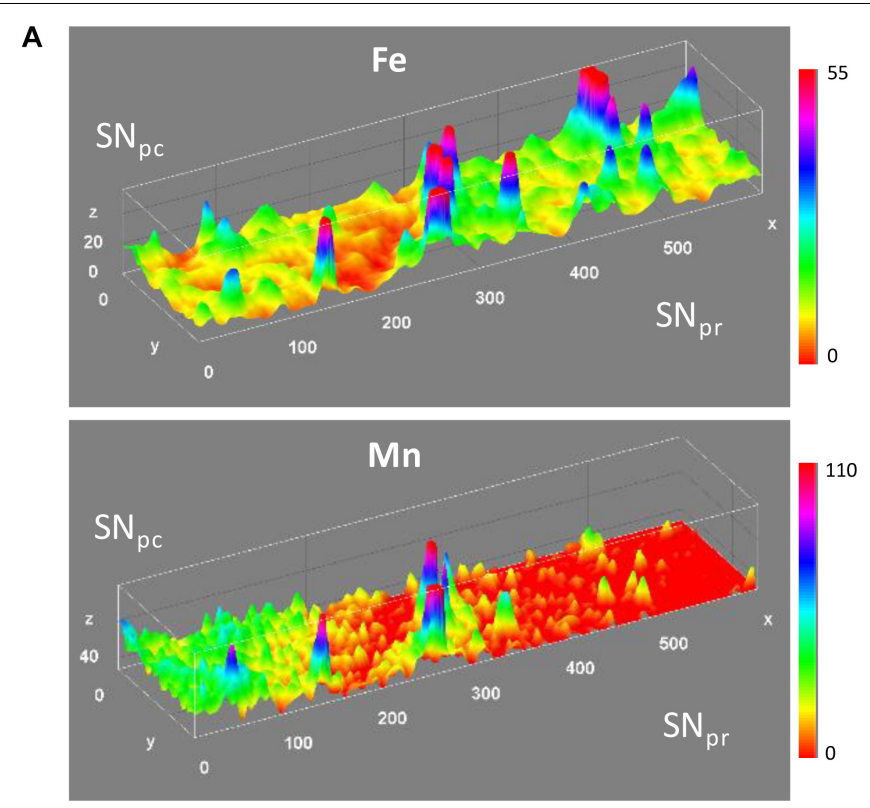

B
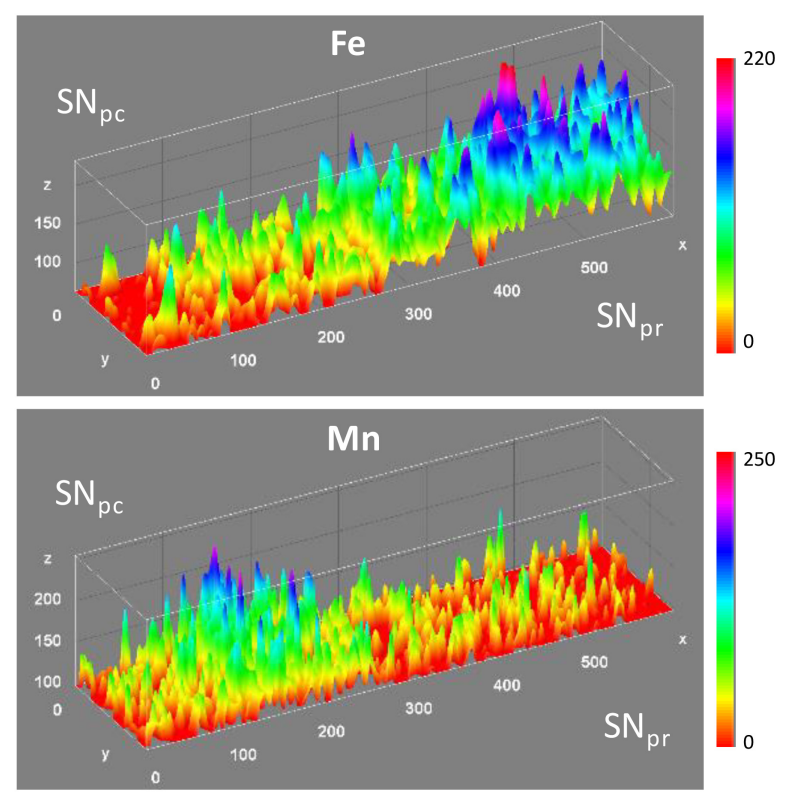

FIGURE 6 | Representative micro-SXRF distribution maps of Fe and Mn in the SNpc, appearing at the left side of the images, and SNpr, right side of the images. (A) Distribution maps of Fe and Mn along the SNpc and SNpr in the ContraL side of sham rat brain. (B) Distribution maps of Fe and Mn in the ContraL side of a 6-OHDA injured rat. Similar distributions were found in other cases with no effect of 6-OHDA. $x$ and $y$ axis in $\mu \mathrm{m}$; $z$ axis in number of $x$-ray fluorescence counts.

Compared to other studies, we used a relatively mild 6OHDA treatment which resulted in a $\mathrm{TH}$ decreased expression ranging from 35 to $85 \%$ (mean $60 \%$ ). These conditions were selected in order to study animals modeling mid-stages of PD progression. However, $\mathrm{Fe}$ increase in the $\mathrm{SN}$ might only be observed following extensive 6-OHDA lesions of the nigrostriatal dopamine pathway, leading to a complete or almost complete depletion of the dopaminergic neurons (Oestreicher et al., 1994; Olmedo-Díaz et al., 2017). In these conditions of complete dopaminergic depletion a $20-30 \%$ increase of total $\mathrm{Fe}$ is generally observed. In our conditions, we were expecting to observe slighter modifications proportional to the level of $\mathrm{TH}$ decrease. Our data indicate that the quantitative modification of Fe content was too low to be observed following mild injections of 6-OHDA. Our data also indicate that the 6OHDA treated rat model of $\mathrm{PD}$ has some limitations to study $\mathrm{Fe}$ dyshomeostasis. Fe accumulation occurs only for very advanced stages of dopamine denervation suggesting that Fe increase might not be involved in the neurodegenerative process induced by 6-OHDA. The 6-OHDA rat model has, however, proven to be very useful to study advanced stages of dopamine neuron degeneration, reproducing some important features observed in $\mathrm{PD}$ such as the increase of $\mathrm{Fe}$ in the $\mathrm{SNpc}$, to study neuroprotective effects of Fe chelators (Dexter et al., 2011). Other investigations have shown that the Fe increase is associated to a decrease in ceruloplasmin expression in the SN of 6-OHDA lesioned-rat (Wang et al., 2015). Ceruloplasmin is a ferroxidase which converts $\mathrm{Fe}$ (II) into $\mathrm{Fe}$ (III) and cooperates with ferroportin 1 to export Fe from cells. Other studies also suggested that the increase of Fe in the SN could be provoked by the dysfunction of the blood brain barrier in the
6-OHDA rat model (Oestreicher et al., 1994; Virel et al., 2014; Olmedo-Díaz et al., 2017).

Since the total Fe content was unchanged following mild injections of 6-OHDA, we investigated if the 6-OHDA treatment could modify the ratio between $\mathrm{Fe}(\mathrm{II}) / \mathrm{Fe}$ (III) in the $\mathrm{SN}$ and be used as an animal model of pro-oxidative conditions related to PD. Some reports using Fe(II) histochemical assays support the Fe oxidation hypothesis in $\mathrm{PD}$, showing the local increase of redox-active $\mathrm{Fe}(\mathrm{II})$ in neuromelanin neurons (Faucheux et al., 2003) and Lewy bodies from the SNpc of PD brains (Castellani et al., 2000). Mixed valences of $\mathrm{Fe}(\mathrm{II}) / \mathrm{Fe}(\mathrm{III})$ have been observed in a single neuron from a PD patient using micro-XANES (Yoshida et al., 2001). There is also one report on animal models, mixed valences of $\mathrm{Fe}(\mathrm{II}) / \mathrm{Fe}$ (III) were observed using micro-XANES on the MPTP primate model of PD showing $\mathrm{Fe}$ (II) and Fe(III) co-localization in neuromelanin neurons (IdeEktessabi and Rabionet, 2005). For all these studies either the Fe histochemistry or the Fe micro-XANES analysis, samples were chemically fixed and analyzed at room temperature. In two other XANES studies where tissues were cryogenically processed, without chemical fixation, there were no significant differences in Fe oxidation state between PD patients and controls, and only Fe(III) was observed (Griffiths et al., 1999; Chwiej et al., 2007). In a study of cryogenically processed tissues from the SN of PD and control subjects, measurements using Mössbauer spectroscopy evidenced the presence of Fe in a ferritin-like form and did not detect any divalent Fe (Wypijewska et al., 2010). Our data obtained in cryogenic native conditions indicate that $\mathrm{Fe}$ is present only as Fe(III) in the 6-OHDA model and XANES spectra from all samples are very similar to ferritin spectrum. This result is in good agreement with XAS and Mössbauer spectroscopy 
data obtained on human PD cryogenically processed tissues (Griffiths et al., 1999; Chwiej et al., 2007; Wypijewska et al., 2010). Overall these results suggest that low temperature sample environment is highly advocated for micro-XAS speciation of $\mathrm{Fe}$, as also suggested by previous studies of biological samples due to their sensitivity to X-ray irradiation (Bacquart et al., 2007; Ortega, 2011; Porcaro et al., 2018). Our data also indicate that $\mathrm{Fe}$ was present exclusively at $\mathrm{Fe}$ (III) oxidation state in all samples, and with a XANES spectral signature similar to that of ferritin. However, the limit of speciation of micro-XANES is in the $10 \mu \mathrm{g} . \mathrm{g}^{-1}$ range (Bacquart et al., 2007) meaning that minor changes in Fe oxidation state or in $\mathrm{Fe}$ (III) speciation, i.e., Fe(III) binding to other molecules than ferritin, would be difficult to evidence with this technique. Other analytical approaches such as electrothermal atomic absorption spectrometry have shown that $\mathrm{Fe}(\mathrm{III})$ could be present within the SN of PD patients in a more labile form than bound to ferritin, with a concentration of labile Fe in the range from 40 to $100 \mathrm{ng}^{-g^{-1}}$ (Wypijewska et al., 2010).

Another important result of this study is the higher content of $\mathrm{Mn}$ in the $\mathrm{SNpc}$, the region rich in dopaminergic neurons, compared to the SNpr. There are some indications in the literature for the potential preferential localization of $\mathrm{Mn}$ in the SNpc vs. the SNpr. A SXRF image of the brain from a rat treated by intraperitoneal injections of $\mathrm{Mn}$ indicated that $\mathrm{Mn}$ content could be higher in the SNpc than in the SNpr (Robison et al., 2012). This result is very similar to our observations of rat brains, but in our case the animals were not treated with $\mathrm{Mn}$. In another study of the same authors, the dopaminergic neurons of the SNpc were identified as potential target of $\mathrm{Mn}$ accumulation after rat exposure to excess Mn (Robison et al., 2015). Mn was clearly detected in the perinuclear region of dopaminergic neurons identified using a retrograde fluorescent tracer. This subcellular distribution is in agreement with data from our group showing the peri-nuclear accumulation of $\mathrm{Mn}$ in the Golgi apparatus of dopamine neurons (Carmona et al., 2010, 2014). Important questions remain open about why and by which mechanism dopaminergic neurons would accumulate preferentially Mn. A higher uptake through the increased expression of the transferrin receptor (Robison et al., 2012) or through a decreased efflux related to the expression of $\mathrm{Mn}$ detoxification proteins such as SLC30A10 (Carmona et al., 2019) are potential mechanisms of $\mathrm{Mn}$ accumulation that still need to be investigated.

In conclusion, metal dyshomeostasis in the nervous system is observed in many neurodegenerative diseases, however, the role of metal modifications in the etiological processes is still not well understood. Micro-analytical approaches are being developed to investigate the dysregulation of biological metals in well-identified regions of the brain. Thanks to the methodology presented in this article, we could investigate the distribution of biological metals in precisely identified brain regions by comparing immuno-histochemical staining and chemical imaging results showing the opposite enrichment of $\mathrm{Fe}$ and $\mathrm{Mn}$ in the SNpr and SNpc, respectively. There are still exciting experiments to perform to fully identify the sites of $\mathrm{Mn}$ and Fe localization in the SNpc and SNpr in normal and pathological conditions. Our data also suggest that the 6-OHDA lesioned rat model, although very helpful to study advanced stages of $\mathrm{PD}$ progression, is limited to investigate intermediate development stages of the disease. The increase of Fe content in the $\mathrm{SN}$ following 6-OHDA administration as reported in the literature is observed when almost all the dopaminergic neurons are lost. Therefore Fe increase in the SN might not be involved in the neurodegenerative process induced by 6-OHDA since we did not observe such Fe dyshomeostasis for intermediate stages of injury (35-85\% of dopaminergic neurons loss). Finally, our study demonstrates the feasibility and details the methodology that could now be applied to investigate other neurodegenerative diseases when metal dyshomeostasis is suspected to be involved.

\section{DATA AVAILABILITY STATEMENT}

The datasets generated for this study are available on request to the corresponding authors.

\section{ETHICS STATEMENT}

The animal study was reviewed and approved by Ethics committee of the Grenoble Institute of Neuroscience.

\section{AUTHOR CONTRIBUTIONS}

AC, MS, and RO contributed to the conception and design of the study. LP and CC prepared samples. CC performed cryo-sections and immunostaining. AC, SR, LP, and RO performed PIXE experiments. AC performed PIXE data treatment. LP, DV, and RO performed synchrotron experiments. DV and RO performed synchrotron data treatment. AC and SR performed the statistical analysis. AC and RO wrote the first draft of the manuscript. All authors contributed to the manuscript revision, and read and approved the submitted version.

\section{FUNDING}

This work was supported by the project NANOCHEMCELL ANR-09-PIRI-0029 of the French National Research Agency (ANR), by CNRS/IN2P3 master project IFI, and by "Osez l'interdisciplinarité" CNRS program.

\section{ACKNOWLEDGMENTS}

We are grateful to AIFIRA Facility at CENBG for beamtime allocation. We acknowledge SOLEIL for provision of synchrotron radiation facilities. We would like to thank LUCIA staff and in particular Nicolas Trcera for assistance in using beamline LUCIA. We are very grateful to Jean Luc Guerquin-Kern (Institut Curie, Orsay) and Sylvain Bohic (Grenoble Institute of Neuroscience) for helpful discussions and contribution to other parts of the project. 


\section{REFERENCES}

Bacquart, T., Devès, G., Carmona, A., Tucoulou, R., Bohic, S., and Ortega, R. (2007). Subcellular speciation analysis of trace element oxidation states using synchrotron radiation micro-X-ray absorption near edge structure. Anal. Chem. 79, 7353-7359. doi: 10.1021/ac0711135

Barnham, K. J., and Bush, A. I. (2014). Biological metals and metal-targeting compounds in major neurodegenerative diseases. Chem. Soc. Rev. 43, 67276749. doi: $10.1039 / \mathrm{c} 4 \mathrm{cs} 00138 \mathrm{a}$

Campbell, J. L., Boyd, N. I., Grassi, N., Bonnick, P., and Maxwell, J. A. (2010). The Guelph PIXE software package IV. Nucl. Instrum. Methods Phys. Res. Sect. B 268, 3356-3363. doi: 10.1016/j.nimb.2010. 07.012

Carmona, A., Deves, G., and Ortega, R. (2008). Quantitative micro-analysis of metal ions in subcellular compartments of cultured dopaminergic cells by combination of three ion beam techniques. Anal. Bioanal. Chem. 390, 15851594. doi: 10.1007/s00216-008-1866-6

Carmona, A., Devès, G., Roudeau, S., Cloetens, P., Bohic, S., and Ortega, R. (2010). Manganese accumulates within Golgi apparatus in dopaminergic cells as revealed by synchrotron X-Ray fluorescence nano-imaging. ACS Chem. Neurosci. 1, 194-203. doi: 10.1021/cn900021z

Carmona, A., Roudeau, S., L'Homel, B., Pouzoulet, F., Bonnet-Boissinot, S., Prezado, Y., et al. (2017). Heterogeneous intratumoral distribution of gadolinium nanoparticles within U87 human glioblastoma xenografts unveiled by micro-PIXE imaging. Anal. Biochem. 523, 50-57. doi: 10.1016/j.ab.2017. 02.010

Carmona, A., Roudeau, S., Perrin, L., Veronesi, G., and Ortega, R. (2014). Environmental manganese compounds accumulate as $\mathrm{Mn}$ (II) within the Golgi apparatus of dopamine cells: relationship between speciation, subcellular distribution, and cytotoxicity. Metallomics 6, 822-832. doi: 10.1039/ c4mt00012a

Carmona, A., Zogzas, C. E., Roudeau, S., Porcaro, F., Garrevoet, J., Spiers, K., et al. (2019). SLC30A10 mutation involved in parkinsonism results in manganese accumulation within nano-vesicles of the Golgi apparatus. ACS Chem. Neurosci. 10, 599-609. doi: 10.1021/acschemneuro.8b00451

Castellani, R. J., Siedlak, S. L., Perry, G., and Smith, M. A. (2000). Sequestration of iron by Lewy bodies in Parkinson's disease. Acta Neuropathol. 100, 111-114. doi: $10.1007 / s 004010050001$

Chwiej, J., Adamek, D., Szczerbowska-Boruchowska, M., Krygowska-Wajs, A., Wojcik, S., Falkenberg, G., et al. (2007). Investigations of differences in iron oxidation state inside single neurons from substantia nigra of Parkinson's disease and control patients using the micro-XANES technique. J. Biol. Inorg. Chem. 12, 204-211. doi: 10.1007/s00775-0060179-5

Chwiej, J., Szczerbowska-Boruchowska, M., Lankosz, M., Wojcik, S., Falkenberg, G., Stegowski, Z., et al. (2005). Preparation of tissue samples for X-ray fluorescence microscopy. Spectrochim. Acta Part B 60, 1531-1537. doi: 10.1016/ j.sab.2005.10.002

Collingwood, J. F., and Adams, F. (2017). Chemical imaging analysis of the brain with X-ray methods. Spectrochim. Acta Part B 130, 101-118. doi: 10.1016/j.sab. 2017.02.013

Collingwood, J. F., and Davidson, M. R. (2014). The role of iron in neurodegenerative disorders: insights and opportunities with synchrotron light. Front. Pharmacol. 5:191. doi: 10.3389/fphar.2014.00191

Davies, K. M., Bohic, S., Carmona, A., Ortega, R., Cottam, V., Hare, D. J., et al. (2014). Copper pathology in vulnerable brain regions in Parkinson's disease. Neurobiol. Aging 35, 858-866. doi: 10.1016/j.neurobiolaging.2013. 09.034

Davies, K. M., Hare, D. J., Bohic, S., James, S. A., Billings, J. L., Finkelstein, D. I., et al. (2015). Comparative study of metal quantification in neurological tissue using laser ablation-inductively coupled plasma-mass spectrometry imaging and X-ray fluorescence microscopy. Anal. Chem. 87, 6639-6645. doi: 10.1021/ acs.analchem.5b01454

Dexter, D. T., Statton, S. A., Whitmore, C., Freinbichler, W., Weinberger, P., Tipton, K. F., et al. (2011). Clinically available iron chelators induce neuroprotection in the 6-OHDA model of Parkinson's disease after peripheral administration. J. Neural. Transm. 118, 223-231. doi: 10.1007/s00702-010$0531-3$
Drayer, B., Burger, P., Darwin, R., Riederer, S., Herfkens, R., and Johnson, G. A. (1986). MRI of brain iron. AJR Am. J. Roentgenol. 147, 103-110.

Faucheux, B. A., Martin, M.-E., Beaumont, C., Hauw, J.-J., Agid, Y., and Hirsch, E. C. (2003). Neuromelanin associated redox-active iron is increased in the substantia nigra of patients with Parkinson's disease. J. Neurochem. 86, 11421148. doi: 10.1046/j.1471-4159.2003.01923.x

Favier, M., Carcenac, C., Drui, G., Boulet, S., El Mestikawy, S., and Savasta, M. (2013). High-frequency stimulation of the subthalamic nucleus modifies the expression of vesicular glutamate transporters in basal ganglia in a rat model of Parkinson's disease. BMC Neurosci. 14:152. doi: 10.1186/1471-220214-152

Feise, R. J. (2002). Do multiple outcome measures require p-value adjustment? BMC Med. Res. Methodol. 2:8. doi: 10.1186/1471-2288-2-8

Flank, A.-M., Cauchon, G., Lagarde, P., Bac, S., Janousch, M., Wetter, R., et al. (2006). LUCIA : a microfocus soft XAS beamline. Nucl. Instrum. Meth. B 246, 269-274. doi: 10.1016/j.nimb.2005.12.007

Fox, J., and Bouchet-Valat, M. (2018). Rcmdr: $R$ Commander. R package version $2.4-4$.

Friedman, A., and Galazka-Friedman, J. (2012). The history of the research of iron in parkinsonian substantia nigra. J. Neural. Transm. 119, 1507-1510. doi: 10.1007/s00702-012-0894-8

Griffiths, P. D., Dobson, B. R., Jones, G. R., and Clarke, D. T. (1999). Iron in the basal ganglia in Parkinson's disease. An in vitro study using extended X-ray absorption fine structure and cryo-electron microscopy. Brain 122, 667-673. doi: $10.1093 /$ brain/122.4.667

Guan, X., Xu, X., and Zhang, M. (2017). Region-specific iron measured by MRI as a biomarker for Parkinson's disease. Neurosci. Bull. 33, 561-567. doi: 10.1007/ s12264-017-0138-x

Hackett, M. J., Hollings, A., Caine, S., Bewer, B. E., Alaverdashvili, M., Takechi, R., et al. (2019). Elemental characterisation of the pyramidal neuron layer within the rat and mouse hippocampus. Metallomics 11, 151-165. doi: 10.1039/ c8mt00230d

Hackett, M. J., McQuillan, J. A., El-Assaad, F., Aitken, J. B., Levina, A., Cohen, D. D., et al. (2011). Chemical alterations to murine brain tissue induced by formalin fixation: implications for biospectroscopic imaging and mapping studies of disease pathogenesis. Analyst 136, 2941-2952. doi: 10.1039/ c0an $00269 \mathrm{k}$

Hare, D. J., George, J. L., Grimm, R., Wilkins, S., Adlard, P. A., Cherny, R. A., et al. (2009). Quantitative elemental bio-imaging of $\mathrm{Mn}, \mathrm{Fe}, \mathrm{Cu}$ and $\mathrm{Zn}$ in 6hydroxydopamine induced Parkinsonism mouse models. Metallomics 1, 53-58. doi: $10.1039 / \mathrm{b} 816188 \mathrm{~g}$

Hare, D. J., George, J. L., Grimm, R., Wilkins, S., Adlard, P. A., Cherny, R. A., et al. (2010). Three-dimensional elemental bio-imaging of $\mathrm{Fe}, \mathrm{Zn}, \mathrm{Cu}, \mathrm{Mn}$ and $\mathrm{P}$ in a 6-hydroxydopamine lesioned mouse brain. Metallomics 2, 745-753. doi: $10.1039 / \mathrm{c} 0 \mathrm{mt} 00039 \mathrm{f}$

Hare, D. J., Lei, P., Ayton, S., Roberts, B. R., Grimm, R., George, J. L., et al. (2014). An iron-dopamine index predicts risk of parkinsonian neurodegeneration in the substantia nigra pars compacta. Chem. Sci. 5, 2160-2169. doi: 10.1039/ C3SC $53461 \mathrm{H}$

He, Y., Thong, P. S., Lee, T., Leong, S. K., Shi, C. Y., Wong, P. T., et al. (1996). Increased iron in the substantia nigra of 6-OHDA induced parkinsonian rats: a nuclear microscopy study. Brain Res. 735, 149-153. doi: 10.1016/0006-8993(96) 00313-7

Ide-Ektessabi, A., and Rabionet, M. (2005). The role of trace metallic elements in neurodegenerative disorders: quantitative analysis using XRF and XANES spectroscopy. Anal. Sci. 21, 885-892. doi: 10.2116/analsci.21.885

James, S. A., Myers, D. E., de Jonge, M. D., Vogt, S., Ryan, C. G., Sexton, B. A., et al. (2011). Quantitative comparison of preparation methodologies for X-ray fluorescence microscopy of brain tissue. Anal. Bioanal. Chem. 401, 853-864. doi: 10.1007/s00216-011-4978-3

Jin, Q., Paunesku, T., Lai, B., Gleber, S. C., Chen, S. I., Finney, L., et al. (2017). Preserving elemental content in adherent mammalian cells for analysis by synchrotron-based x-ray fluorescence microscopy. J. Microsci. 265, 81-93. doi: 10.1111/jmi.12466

Liddell, J. R., and White, A. R. (2018). Nexus between mitochondrial function, iron, copper and glutathione in Parkinson's disease. Neurochem. Int. 117, 126-138. doi: 10.1016/j.neuint.2017.05.016 
Mayer, M. (1997). SIMNRA User's Guide, Report IPP 9/113. Garching: Max-PlanckInstitut fur Plasmaphysik.

Morawski, M., Meinecke, C. H., Reinert, T., Dorffel, A. C., Riederer, P., Arendt, T., et al. (2005). Determination of trace elements in the human substantia nigra. Nucl. Instrum. Methods Phys. Res. B 231, 224-228. doi: 10.1016/j.nimb.2005. 01.061

Morris, C. M., and Edwardson, J. A. (1994). Iron histochemistry of the substantia nigra in Parkinson's disease. Neurodegeneration 3, 277-282.

Oakley, A. E., Collingwood, J. F., Dobson, J., Love, G., Perrott, H. R., Edwardson, J. A., et al. (2007). Individual dopaminergic neurons show raised iron levels in Parkinson disease. Neurology 68, 1820-1825. doi: 10.1212/01.wnl.0000262033. 01945.9a

Oestreicher, E., Sengstock, G. J., Riederer, P., Olanow, C. W., Dunn, A. J., and Arendash, G. W. (1994). Degeneration of nigrostriatal dopaminergic neurons increases iron within the substantia nigra: a histochemical and neurochemical study. Brain Res. 660, 8-18. doi: 10.1016/0006-8993(94)90833-8

Olmedo-Díaz, S., Estévez-Silva, H., Orädd, G., Af Bjerkén, S., Marcellino, D., and Virel, A. (2017). An altered blood-brain barrier contributes to brain iron accumulation and neuroinflammation in the 6-OHDA rat model of Parkinson's disease. Neuroscience 362, 141-151. doi: 10.1016/j.neuroscience.2017. 08.023

Ortega, R. (2011). Direct speciation analysis of inorganic elements in single cells using X-ray absorption spectroscopy. J. Anal. Atomic Spectrom. 26, 23-29. doi: 10.1039/C0JA00153H

Ortega, R., Carmona, A., Llorens, I., and Solari, P. L. (2012). X-ray absorption spectroscopy of biological samples. A tutorial. J. Anal. Atomic Spectrom. 27, 2054-2065. doi: 10.1039/C2JA30224A

Ortega, R., Devès, G., and Carmona, A. (2009). Bio-metals imaging and speciation in cells using proton and synchrotron radiation X-ray micro-spectroscopy. J. $R$. Soc. Interface 6, S649-S658. doi: 10.1098/rsif.2009.0166.focus

Paxinos, G., and Watson, C. (2007). The Rat Brain in Stereotaxic Coordinates. London: Academic Press.

Perrin, P., Carmona, A., Roudeau, S., and Ortega, R. (2015). Evaluation of sample preparation methods for single cell quantitative elemental imaging using proton or synchrotron radiation focused beams. J. Anal. Atomic Spectrom. 30:2525. doi: 10.1039/C5JA00303B

Popescu, B. F., George, M. J., Bergmann, U., Garachtchenko, A. V., Kelly, M. E., McCrea, R. P., et al. (2009). Mapping metals in Parkinson's and normal brain using rapid-scanning x-ray fluorescence. Phys. Med. Biol. 54, 651-663. doi: 10.1088/0031-9155/54/3/012

Porcaro, F., Roudeau, S., Carmona, A., and Ortega, R. (2018). Advances in element speciation analysis of biomedical samples using synchrotron-based techniques. Trends Anal. Chem. 104, 22-41. doi: 10.1016/j.trac.2017.09.016

Pushie, M. J., Pickering, I. J., Korbas, M., Hackett, M. J., and George, G. N. (2014). Elemental and chemically specific X-ray fluorescence imaging of biological systems. Chem. Rev. 114, 8499-8541. doi: 10.1021/cr4007297

R Core Team (2013). R: A Language and Environment for Statistical Computing. Vienna: R Foundation for Statistical Computing.

Ralle, M., and Lutsenko, S. (2009). Quantitative imaging of metals in tissues. Biometals 22, 197-205. doi: 10.1007/s10534-008-9200-5

Riederer, P., Sofic, E., Rausch, W.-D., Schmidt, B., Reynolds, G. P., Jellinger, K., et al. (1989). Transition metals, ferritin, glutathione, and ascorbic acid in parkinsonian brains. J. Neurochem. 52, 515-520. doi: 10.1111/j.1471-4159.1989. tb09150.x

Robison, G., Sullivan, B., Cannon, J. R., and Pushkar, Y. (2015). Identification of dopaminergic neurons of the substantia nigra pars compacta as a target of manganese accumulation. Metallomics 7, 748-755. doi: 10.1039/c5mt00023h

Robison, G., Zakharova, T., Fu, S., Jiang, W., Fulper, R., Barrea, R., et al. (2012). $\mathrm{X}$-ray fluorescence imaging: a new tool for studying manganese neurotoxicity. PLoS One 7:e48899. doi: 10.1371/journal.pone.0048899
Roudeau, S., Carmona, A., Perrin, L., and Ortega, R. (2014). Correlative organelle fluorescence microscopy and synchrotron X-ray chemical element imaging in single cells. Anal. Bioanal. Chem. 406, 6979-6991. doi: 10.1007/s00216-0148004-4

RStudio Team (2016). RStudio: Integrated Development for R. Boston, MA: RStudio, Inc.

Sian-Hülsmann, J., Mandel, S., Youdim, M. B., and Riederer, P. (2011). The relevance of iron in the pathogenesis of Parkinson's disease. J. Neurochem. 118, 939-957. doi: 10.1111/j.1471-4159.2010.07132.x

Snyder, A. M., and Connor, J. R. (2009). Iron, the substantia nigra and related neurological disorders. Biochim. Biophys. Acta 1790, 606-614. doi: 10.1016/j. bbagen.2008.08.005

Sofic, E., Riederer, P., Heinsen, H., Beckmann, H., Reynolds, G. P., Hebenstreit, G., et al. (1988). Increased iron (III) and total iron content in post mortem substantia nigra of parkinsonian brain. J. Neural. Transm. 74, 199-205. doi: $10.1007 / \mathrm{bf} 01244786$

Toni, M., Massimino, M. L., De Mario, A., Angiulli, E., and Spisni, E. (2017). Metal dyshomeostasis and their pathological role in prion and prion-like diseases: the basis for a nutritional approach. Front. Neurosci. 11:3. doi: 10.3389/fnins.2017. 00003

Vantelon, D., Trcera, N., Roy, D., Moreno, T., Mailly, D., Guilet, S., et al. (2016). The LUCIA beamline at SOLEIL. J. Synchrotron. Rad. 23, 635-640. doi: 10.1107/ S1600577516000746

Virel, A., Faergemann, E., Orädd, G., and Strömberg, I. (2014). Magnetic resonance imaging (MRI) to study striatal iron accumulation in a rat model of Parkinson's disease. PLoS One 9:e112941. doi: 10.1371/journal.pone.0112941

Wang, J., Bi, M., and Xie, J. (2015). Ceruloplasmin is involved in the nigral iron accumulation of 6-OHDA-lesioned rats. Cell. Mol. Neurobiol. 35, 661-668. doi: 10.1007/s10571-015-0161-2

Wang, J., Jiang, H., and Xie, J. X. (2004). Time dependent effects of 6OHDA lesions on iron level and neuronal loss in rat nigrostriatal system. Neurochem. Res. 29, 2239-2243. doi: 10.1007/s11064-0047031-5

Ward, R. J., Zucca, F. A., Duyn, J. H., Crichton, R. R., and Zecca, L. (2014). The role of iron in brain ageing and neurodegenerative disorders. Lancet Neurol. 13, 1045-1060. doi: 10.1016/S1474-4422(14)70 $117-6$

Watt, F., Lee, T., Thong, P. S. P., and Tang, S. M. (1995). Nuclear microscopy in Parkinson's disease. Nucl. Instrum. Methods B 104, 361-364. doi: 10.1016/0168583X(95)00455-6

Wypijewska, A., Galazka-Friedman, J., Bauminger, E. R., Wszolek, Z. K., Schweitzer, K. J., Dickson, D. W., et al. (2010). Iron and reactive oxygen species activity in parkinsonian substantia nigra Parkinsonism Relat. Disord. 16, 329-333. doi: 10.1016/j.parkreldis.2010. 02.007

Yoshida, S., Ide-Ektessabi, A., and Fujisawa, S. (2001). XANES spectroscopy of a single neuron from a patient with Parkinson's disease. J. Synchrotron Radiat. 8, 998-1000. doi: 10.1107/s0909049500017726

Conflict of Interest: The authors declare that the research was conducted in the absence of any commercial or financial relationships that could be construed as a potential conflict of interest.

Copyright (c) 2019 Carmona, Roudeau, Perrin, Carcenac, Vantelon, Savasta and Ortega. This is an open-access article distributed under the terms of the Creative Commons Attribution License (CC BY). The use, distribution or reproduction in other forums is permitted, provided the original author(s) and the copyright owner(s) are credited and that the original publication in this journal is cited, in accordance with accepted academic practice. No use, distribution or reproduction is permitted which does not comply with these terms. 